\title{
Reliable random error estimation in the measurement of line-strength indices
}

\author{
N. Cardiel ${ }^{1}$, J. Gorgas ${ }^{1}$, J. Cenarro ${ }^{1}$, and J.J. González ${ }^{2}$ \\ 1 Departamento de Astrofísica, Facultad de Físicas, Universidad Complutense, 28040 Madrid, Spain \\ 2 Instituto de Astronomía, U.N.A.M., Apdo. Postal 70-264, 04510 México D.F., México
}

Received April 24; accepted June 17, 1997

\begin{abstract}
We present a new set of accurate formulae for the computation of random errors in the measurement of atomic and molecular line-strength indices. The new expressions are in excellent agreement with numerical simulations. We have found that, in some cases, the use of approximated equations can give misleading line-strength index errors. It is important to note that accurate errors can only be achieved after a full control of the error propagation throughout the data reduction with a parallel processing of data and error images. Finally, simple recipes for the estimation of the required signal-to-noise ratio to achieve a fixed index error are presented.
\end{abstract}

Key words: methods: data analysis - methods: analytical — methods: statistical

\section{Introduction}

Line-strength indices have proven to be excellent tools for the study of absorption features in the spectra of astronomical objects. In particular, since the pioneering work of Faber (1973), the behaviour of indices in composite stellar systems has supplied fundamental clues for understanding the history of their stellar populations (e.g. Burstein et al. 1984; Bica et al. 1990; Gorgas et al. 1990; Worthey et al. 1992; Bender et al. 1993; Jones \& Worthey 1995; Davies 1996). At the light of up-to-date stellar population models, line-strength indices can provide constrains about important parameters such as mean age, metallicity, or abundance ratios (e.g. Worthey 1994; Vazdekis et al. 1996; Bressan et al. 1996). In addition, linestrength indices have been widely employed to determine basic stellar atmospheric parameters (Rich 1988; Zhou 1991; Terndrup et al. 1995) and star cluster abundances (Brodie \& Hanes 1986; Mould et al. 1990; Gregg 1994; Minniti 1995; Huchra et al. 1996), as well as to confront cosmological issues by providing new distance indicators (Dressler et al. 1987) and a powerful tool to investigate the redshift evolution of galaxies (Hamilton 1985; Charlot \& Silk 1994; Bender et al. 1996).

Obviously, the suitability of line-strength indices to investigate the above items relies on a proper determination of the associated index errors. For illustration, it is interesting to note how errors in the measurement of absorption features translate into uncertainties in the derived mean age and metallicity of an old stellar population. Taking the predictions of the single-burst stellar population models of Worthey (1994) as a reference, typical errors of $0.20 \AA$ in the Lick indices Fe 5270 and $\mathrm{H} \beta$ (see below) translate into $\Delta[\mathrm{Fe} / \mathrm{H}] \simeq 0.18$ dex and $\Delta$ age $\simeq 6$ Gyr respectively (for a composite population of $10 \mathrm{Gyr}$ and metallicity around $[\mathrm{Fe} / \mathrm{H}]=0.3)$. Another example in which it is extremely important to obtain reliable index errors is the analysis of the intrinsic scatter of relations such as that of $\mathrm{Mg}_{2}$ with velocity dispersion in elliptical galaxies (Schweizer et al. 1990; Bender et al. 1993).

An accurate error determination is therefore needed in order to draw confident interpretations from observed data. Although this requires the estimation of both random and systematic errors, in this paper we will concentrate in the former. Whereas random errors can be readily derived by applying statistical methods, systematic errors do not always allow such a straight approach. Several authors have dealt with the most common sources of systematic errors in the measurement of line-strength indices. These include: flux calibration effects (González 1993; Davies et al. 1993; Cardiel et al. 1995), spectral resolution and velocity dispersion corrections (Gorgas et al. 1990; González 1993; Carollo et al. 1993; Davies et al. 1993; Fisher et al. 1996; Vazdekis et al. 1997), sky subtraction uncertainties (Saglia et al. 1993; Davies et al. 1993; Cardiel et al. 1995; Fisher et al. 1995), scatter light effects (González 1993), wavelength calibration and radial velocity errors (Cardiel et al. 1995; Vazdekis et al. 1997), seeing and focus corrections (Thomsen \& Baum 1987; González 1993; Fisher et al. 1995), deviations from linearity response of the detectors (Gorgas et al. 1990; Cardiel 
et al. 1995), and contamination by nebular emission lines (González 1993; Goudfrooij \& Emsellem 1996).

Although it is always possible to obtain estimates of index errors by performing multiple observations of the same object, analytical formulae to avoid such an observing time effort are clearly needed. In this paper we present a set of analytical expressions to derive reliable errors in the measurement of line-strength indices (hereafter we use error to quote random errors exclusively). General index definitions are given in Sect. 2. Section 3 describes how a proper treatment of error propagation throughout data reduction is a prerequisite to compute confident index errors. Random errors can be obtained through numerical simulations, presented in Sect. 4, or analytically. Previous works are briefly reviewed in Sect. 5, with special emphasis to the approach followed by González (1993). Our final set of formulae is presented in Sect. 6. Section 7 gives some recipes to estimate the required signal-to-noise ratios to achieve a fixed index error.

\section{Definitions}

The strengths of absorption spectral features have been measured in different ways so far. However, although with slight differences among them, most authors have employed line-strength indices with definitions close to the classical expression for an equivalent width:

$W_{\lambda}(\AA)=\int_{\text {line }}(1-S(\lambda) / C(\lambda)) \mathrm{d} \lambda$

where $S(\lambda)$ is the observed spectrum and $C(\lambda)$ is the local continuum usually obtained by interpolation of $S(\lambda)$ between two adjacent spectral regions (e.g. Faber 1973; Faber et al. 1977; Whitford \& Rich 1983; Gorgas 1987; Brodie \& Huchra 1990; González 1993; Rose 1994). In order to avoid subjective determinations of the local continuum, line-strength indices following Eq. (1), referred as atomic indices, are completely characterized by three wavelength regions (bandpasses). The spectral feature of interest is covered by the central bandpass whereas the other two bandpasses, located towards the red and blue of the central region, are employed to define the continuum reference level through a linear interpolation. As pointed out by Geisler (1984) (see also Rich 1988), at low spectral resolution a pseudo-continuum is measured instead of a true continuum.

Line-strength indices are sometimes measured in magnitudes using:

$I(\mathrm{mag})=-2.5 \log _{10}\left(1-\frac{W_{\lambda}(\AA)}{\Delta \lambda}\right)$

where $\Delta \lambda$ is the width of the central bandpass. These line-strengths, referred as molecular indices since they are used for molecular-band features, are defined with the help of broad bandpasses with the continuum regions located far from the central feature. On the other hand, atomic indices, which measure the absorption of atomic spectral features, have narrower and neighboring bandpasses.

Throughout this paper we use the definitions given by González (1993), in which atomic $\left(I_{\mathrm{a}}\right)$ and molecular $\left(I_{\mathrm{m}}\right)$ indices are defined as follows:

$$
\begin{gathered}
I_{\mathrm{a}} \equiv \int_{\lambda_{c_{1}}}^{\lambda_{c_{2}}}(1-S(\lambda) / C(\lambda)) \mathrm{d} \lambda \\
I_{\mathrm{m}} \equiv-2.5 \log _{10} \frac{\int_{\lambda_{c_{1}}}^{\lambda_{c_{2}}} S(\lambda) / C(\lambda) \mathrm{d} \lambda}{\lambda_{c_{2}}-\lambda_{c_{1}}}
\end{gathered}
$$

where $\lambda_{c_{1}}$ and $\lambda_{c_{2}}$ are the limits of the central bandpass (in $\AA$ ). The local pseudo-continuum $C(\lambda)$ is derived by

$C(\lambda) \equiv S_{b} \frac{\lambda_{r}-\lambda}{\lambda_{r}-\lambda_{b}}+S_{r} \frac{\lambda-\lambda_{b}}{\lambda_{r}-\lambda_{b}} \quad$ where

$S_{b} \equiv \frac{\int_{\lambda_{b_{1}}}^{\lambda_{b_{2}}} S(\lambda) \mathrm{d} \lambda}{\left(\lambda_{b_{2}}-\lambda_{b_{1}}\right)}, \quad S_{r} \equiv \frac{\int_{\lambda_{r_{1}}}^{\lambda_{r_{2}}} S(\lambda) \mathrm{d} \lambda}{\left(\lambda_{r_{2}}-\lambda_{r_{1}}\right)}$

$\lambda_{b} \equiv\left(\lambda_{b_{1}}+\lambda_{b_{2}}\right) / 2, \quad \lambda_{r} \equiv\left(\lambda_{r_{1}}+\lambda_{r_{2}}\right) / 2$

being $\lambda_{b_{1}}, \lambda_{b_{2}}, \lambda_{r_{1}}$, and $\lambda_{r_{2}}$ the limits of the blue and red bandpasses respectively.

Although simplified versions of these expressions (i.e. considering a constant continuum flux along the central bandpass, or replacing the integrals by mean values) yield similar results at intermediate resolution, the more accurate formulae must be favoured in order to guarantee the comparisons with high-resolution high- $S / N$ (signalto-noise ratio) spectra (specially for asymmetric indices, as already noted by Worthey et al. 1994).

Probably, the most widely index definition system employed so far is that established by the Lick group (Burstein et al. 1984, 1986; Faber et al. 1985; Gorgas et al. 1993; Worthey et al. 1994). In Table 1 we list the exact definitions (as given by Trager 1997) for the 21 indices which constitute the extended Lick system (see also González 1993). This table also includes the definitions given by Díaz et al. (1989) for the CaII triplet in the nearinfrared. In this paper we will concentrate on the analysis of line-strength errors for these particular indices, although the derived analytical expressions are valid for any general index following Eqs. (3) or (4).

Another interesting spectral feature which will be studied in this paper is the amplitude of the $\lambda 4000-\AA$ break $\left(D_{4000}\right)$. We adopt here the definition given by Bruzual (1983):

$D_{4000} \equiv \frac{\int_{4050}^{4250} S(\nu) \mathrm{d} \lambda}{\int_{3750}^{3950} S(\nu) \mathrm{d} \lambda}$.

This index can be considered as a pseudo-color, being the combination of $S(\nu)$ and $\mathrm{d} \lambda$ due to historical reasons. 
Table 1. Bandpass definitions for the Lick index system - using the revised bandpass limits as given by Trager (1997) -, together with the CaII triplet according to Díaz et al. (1989). The $S / N$ constants $c_{1}, c_{2}$ and $c_{3}$ are explained in Sect. 7

\begin{tabular}{|c|c|c|c|c|}
\hline Index Name & Central Bandpass $(\AA)$ & Continuum Bandpasses $(\AA)$ & \multicolumn{2}{|c|}{$c_{i}$} \\
\hline \multicolumn{3}{|c|}{ Atomic Indices } & $c_{1}$ & $c_{2}$ \\
\hline $\mathrm{Ca} 4227$ & $4222.250-4234.750$ & $\begin{array}{l}4211.000-4219.750 \\
4241.000-4251.000\end{array}$ & 4.604 & 0.3684 \\
\hline G4300 & $4281.375-4316.375$ & $\begin{array}{l}4266.375-4282.625 \\
4318.875-4335.125\end{array}$ & 8.537 & 0.2439 \\
\hline $\mathrm{Fe} 4383$ & $4369.125-4420.375$ & $\begin{array}{l}4359.125-4370.375 \\
4442.875-4455.375\end{array}$ & 13.220 & 0.2580 \\
\hline $\mathrm{Ca} 4455$ & $4452.125-4474.625$ & $\begin{array}{l}4445.875-4454.625 \\
4477.125-4492.125\end{array}$ & 7.038 & 0.3128 \\
\hline $\mathrm{Fe} 4531$ & $4514.250-4559.250$ & $\begin{array}{l}4504.250-4514.250 \\
4560.500-4579.250\end{array}$ & 11.299 & 0.2511 \\
\hline Fe4668 & $4634.000-4720.250$ & $\begin{array}{l}4611.500-4630.250 \\
4742.750-4756.500\end{array}$ & 17.757 & 0.2059 \\
\hline $\mathrm{H} \beta$ & $4847.875-4876.625$ & $\begin{array}{l}4827.875-4847.875 \\
4876.625-4891.625\end{array}$ & 7.301 & 0.2539 \\
\hline Fe5015 & $4977.750-5054.000$ & $\begin{array}{l}4946.500-4977.750 \\
5054.000-5065.250\end{array}$ & 16.455 & 0.2158 \\
\hline $\mathrm{Mg}_{b}$ & $5160.125-5192.625$ & $\begin{array}{l}5142.625-5161.375 \\
5191.375-5206.375\end{array}$ & 8.032 & 0.2472 \\
\hline Fe5270 & $5245.650-5285.650$ & $\begin{array}{l}5233.150-5248.150 \\
5285.650-5318.150\end{array}$ & 9.250 & 0.2313 \\
\hline Fe5335 & $5312.125-5352.125$ & $\begin{array}{l}5304.625-5315.875 \\
5353.375-5363.375\end{array}$ & 10.741 & 0.2685 \\
\hline Fe5406 & $5387.500-5415.000$ & $\begin{array}{l}5376.250-5387.500 \\
5415.000-5425.000\end{array}$ & 7.256 & 0.2893 \\
\hline Fe5709 & $5696.625-5720.375$ & $\begin{array}{l}5672.875-5696.625 \\
5722.875-5736.625\end{array}$ & 6.362 & 0.2679 \\
\hline Fe5782 & $5776.625-5796.625$ & $\begin{array}{l}5765.375-5775.375 \\
5797.875-5811.625\end{array}$ & 6.134 & 0.3067 \\
\hline $\mathrm{NaD}$ & $5876.875-5909.375$ & $\begin{array}{l}5860.625-5875.625 \\
5922.125-5948.125\end{array}$ & 8.113 & 0.2496 \\
\hline Ca1 & $8483.000-8513.000$ & $\begin{array}{l}8447.500-8462.500 \\
8842.500-8857.500\end{array}$ & 8.852 & 0.2951 \\
\hline $\mathrm{Ca} 2$ & $8527.000-8557.000$ & $\begin{array}{l}8447.500-8462.500 \\
8842.500-8857.500\end{array}$ & 8.330 & 0.2777 \\
\hline $\mathrm{Ca} 3$ & $8647.000-8677.000$ & $\begin{array}{l}8447.500-8462.500 \\
8842.500-8857.500\end{array}$ & 7.750 & 0.2583 \\
\hline \multicolumn{3}{|c|}{ Molecular Indices } & \multicolumn{2}{|c|}{$c_{3}$} \\
\hline $\mathrm{CN}_{1}$ & $4142.125-4177.125$ & $\begin{array}{l}4080.125-4117.625 \\
4244.125-4284.125\end{array}$ & \multicolumn{2}{|c|}{0.2241} \\
\hline $\mathrm{CN}_{2}$ & $4142.125-4177.125$ & $\begin{array}{l}4083.875-4096.375 \\
4244.125-4284.125\end{array}$ & \multicolumn{2}{|c|}{0.2691} \\
\hline $\mathrm{Mg}_{1}$ & $5069.125-5134.125$ & $\begin{array}{l}4895.125-4957.625 \\
5301.125-5366.125\end{array}$ & \multicolumn{2}{|c|}{0.1662} \\
\hline $\mathrm{Mg}_{2}$ & $5154.125-5196.625$ & $\begin{array}{l}4895.125-4957.625 \\
5301.125-5366.125\end{array}$ & \multicolumn{2}{|c|}{0.1933} \\
\hline $\mathrm{TiO}_{1}$ & $5936.625-5994.125$ & $\begin{array}{l}5816.625-5849.125 \\
6038.625-6103.625\end{array}$ & \multicolumn{2}{|c|}{0.1824} \\
\hline $\mathrm{TiO}_{2}$ & $6189.625-6272.125$ & $\begin{array}{l}6066.625-6141.625 \\
6372.625-6415.125 \\
\end{array}$ & \multicolumn{2}{|c|}{0.1568} \\
\hline
\end{tabular}




\section{Propagation of errors throughout data reduction}

The aim of the reduction process is to minimize the influence of data acquisition imperfections on the estimation of the desired astronomical quantity (see Gilliland 1992 for a short review on noise sources and reduction processes of CCD data). For this purpose, one must perform appropriate manipulations with the data and calibration frames. The arithmetic work involved in this process must be taken into account in order to get reliable estimates of line-strength errors.

In order to trace in full detail the error propagation, error frames must be created at the beginning of the reduction procedure. After this point, error and data frames should be processed in parallel, translating the basic arithmetic manipulations performed over the data images into the error frames by following the law of combination of errors.

The starting point is the creation of initial error images with the expected rms variances from photon statistics and read-out noise. For a single spectrum:

$\sigma^{2}[j]=\frac{1}{g} N_{\mathrm{c}}[j]+\sigma_{\mathrm{RN}}^{2}[j]$

where $\sigma^{2}[j]$ is the variance in the pixel $[j](\sigma[j]$ in number of counts, ADU, - analogic to digital number-), $g$ the gain of the A/D converter (in $\left.\mathrm{e}^{-} / \mathrm{ADU}\right), N_{\mathrm{c}}[j]$ the number of counts in the pixel $[j]$ (after the bias-level subtraction), and $\sigma_{\mathrm{RN}}[j]$ is the read-out noise (in ADU) ${ }^{1}$.

Some of the reduction steps that may have a non negligible effect in the index errors are flatfielding, geometrical distortion corrections, wavelength calibration, sky subtraction and rebinning of the spectra. Note that if error spectra were computed from the final number of counts in the reduced data frame, index errors would tend to be underestimated. The extra benefit of a parallel processing of data and error frames is the possibility of obtaining, at any time of the reduction, the variation in the mean $S / N$ ratio produced by a particular reduction step. Under these conditions, it is possible to determine which parts of the reduction process are more sensitive to errors and even to decide whether some manipulations of the data images can be avoided, either because the resulting $S / N$ ratio is seriously reduced and the benefit of the product insignificant, or because such manipulations become a waste of time.

A full error propagation in the reduction of spectroscopic data has been previously implemented by González

\footnotetext{
1 Note that the apparent dimensional inconsistency of Eq. (9) is not real, and arises from the fact that one of the properties of the Poisson distribution is that its variance is numerically equal to the mean expected number of events.
}

(1993), and it is also included in the reduction package $\mathrm{RED}_{\mathrm{m}}^{\mathrm{uc}} \mathrm{E}$ (Cardiel \& Gorgas 1997) ${ }^{2}$.

To obtain errors on line-strength indices from the reduced data and error spectra, two different approaches can be followed. On one hand, analytical formulae to evaluate index errors as a function of the data and variance values in each pixel can be applied (González 1993). Another method is to simulate numerically the effect of the computed pixel variances in the index measurements (e.g. Cardiel et al. 1995). Both techniques are examined in the next sections.

\section{Numerical simulations}

The effect of random noise in the spectra of astronomical objects can be simulated by introducing in each pixel Gaussian noise computed as:

$\mathcal{R}[j]=\sqrt{2} \times \sigma[j] \sqrt{-\ln \left(1-r_{1}\right)} \cos \left(2 \pi r_{2}\right)$

where $\sigma^{2}[j]$ is the variance in the pixel $[j]$, and $r_{1}$ and $r_{2}$ two random numbers in the range $r_{1}, r_{2} \in[0,1)$. After the creation of $N_{\text {simul }}$ synthetic spectra, index errors can be derived as the unbiased standard deviation of the $N_{\text {simul }}$ measurements of each index (typically we employed $N_{\text {simul }} \sim 1000$ )

For illustration, we show the results of numerical simulations using a high $S / N$ ratio spectrum of the bright star HR 3428 as a template. We have assumed this spectrum to be noiseless, i.e. the nominal values of the measured line-strength indices in this spectrum were considered as error free reference values. By dividing this template spectrum by different constant factors, we built a set of synthetic error spectra which were used to derive index errors as a function of the mean $(S / N$-ratio) $/ \AA$ for different atomic and molecular indices (Fig. 1). In a logarithmic scale, there is a clear linear correlation between the estimated relative error and the $(S / N$-ratio $) / \AA$. In addition, and as it should be expected, at a fixed $(S / N$-ratio $) / \AA$ relative errors for atomic indices (with narrow bandpasses) are larger than for molecular indices.

\section{Analytical formulae: Previous works}

Several authors have employed analytical formulae to estimate errors in the measurement of line-strength indices (Rich 1988; Brodie \& Huchra 1990; Carollo et al. 1993; González 1993). However, the derived error expressions have always been obtained from approximated versions of Eqs. (3) and (4), in which integrals are replaced by averaged number of counts in the bandpasses. Although this approximation works properly under certain circumstances, some additional assumed simplifications seriously constrain the suitability of such formulae. The expressions

Available at:

http://www.ucm.es/OTROS/Astrof/reduceme/reduceme.html 


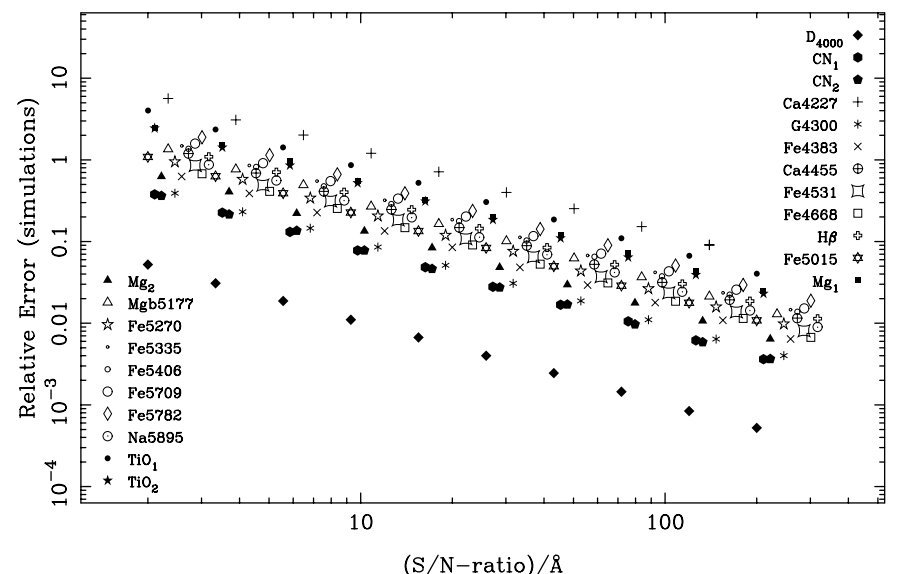

Fig. 1. Relative errors from numerical simulations in the measurement of 21 line-strength indices in the bright star HR 3428, as a function of the mean $S / N$-ratio per $\AA$

derived by Rich (1988) do not take into account readout noise (which can be important at low count levels) nor the effect of the sky subtraction. In the formulae employed by Brodie \& Huchra (1990) and Carollo et al. (1993), although these factors are considered, important reduction steps such as flatfielding (in particular slit illumination) are not taken into account. All these problems are readily settled with a parallel reduction of data and error frames (González 1993, Sect. 3 this paper). Although this approach requires a more elaborate reduction, it yields the most confident results since it allows the application of analytical formulae which consider the final error in each pixel of the reduced spectra.

The most accurate set of analytic formulae published so far for the computation of errors in line-strength indices are those presented by González (1993). As a reference, we reproduce here his equations:

$$
\begin{aligned}
& \sigma\left[I_{\mathrm{a}}\right]= \\
& \frac{S_{c}}{C_{c}} \sqrt{\left(\frac{\sigma_{S_{c}}}{S_{c}}\right)^{2}+\frac{\sigma_{S_{b}}^{2}}{C_{c}^{2}}\left(\frac{\lambda_{r}-\lambda_{c}}{\lambda_{r}-\lambda_{b}}\right)^{2}+\frac{\sigma_{S_{r}}^{2}}{C_{c}^{2}}\left(\frac{\lambda_{b}-\lambda_{c}}{\lambda_{r}-\lambda_{b}}\right)^{2}}
\end{aligned}
$$

$\sigma\left[I_{\mathrm{m}}\right]=\frac{2.5 \times 10^{0.4 I_{\mathrm{m}}}}{2.3026\left(\lambda_{c_{2}}-\lambda_{c_{1}}\right)} \sigma\left[I_{\mathrm{a}}\right]$

where

$$
\begin{aligned}
\lambda_{c} & \equiv\left(\lambda_{c_{1}}+\lambda_{c_{2}}\right) / 2 \\
C_{c} & \equiv C\left(\lambda_{c}\right) \\
S_{c} & \equiv \int_{\lambda_{c_{1}}}^{\lambda_{c_{2}}} S(\lambda) \mathrm{d} \lambda \\
\left(\sigma_{S_{c}} / S_{c}\right)^{2} & \equiv 1 / \int_{\lambda_{c_{1}}}^{\lambda_{c_{2}}} \frac{S^{2}(\lambda)}{\sigma^{2}(\lambda)} \mathrm{d} \lambda
\end{aligned}
$$

$$
\begin{aligned}
\sigma_{S_{b}}^{2} & \equiv S_{b}^{2} / \int_{\lambda_{b_{1}}}^{\lambda_{b_{2}}} \frac{S^{2}(\lambda)}{\sigma^{2}(\lambda)} \mathrm{d} \lambda \\
\sigma_{S_{r}}^{2} & \equiv S_{r}^{2} / \int_{\lambda_{r_{1}}}^{\lambda_{r_{2}}} \frac{S^{2}(\lambda)}{\sigma^{2}(\lambda)} \mathrm{d} \lambda
\end{aligned}
$$

and $\sigma^{2}(\lambda)$ is the variance of $S(\lambda)$ at the wavelength $\lambda$.

In order to check the accuracy of Eqs. (11) and (12) we compared the predictions of such expressions with the results obtained from numerical simulations. We took spectra and associated error spectra of a large homogeneous sample of 350 standard stars from the Lick library (observed with RBS - Richardson Brealy Spectrograph - at the JKT - Jacobus Kapteyn Telescope - of the Roque de los Muchachos Observatory, La Palma, February 1995). The error spectra were obtained by following a parallel reduction of data and error frames, as it has been previously described. As it is apparent from Fig. 2, there is an excellent agreement between González's formulae and the simulations.

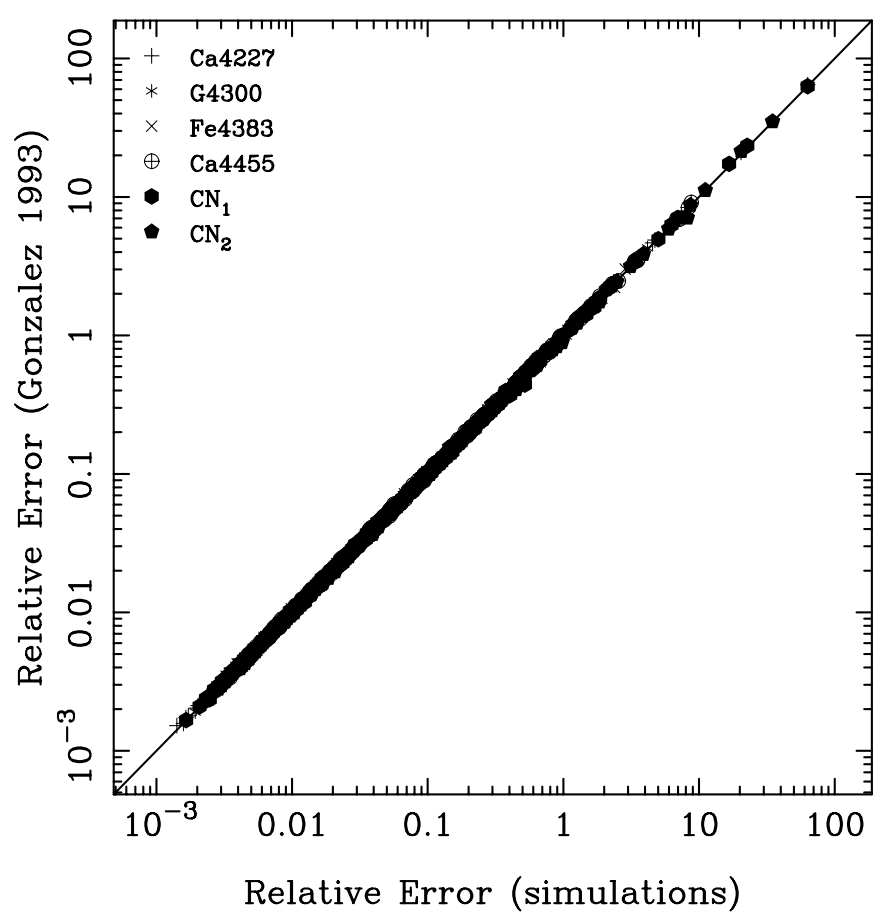

Fig. 2. Comparison of the relative errors employing González's formulae with those obtained from numerical simulations. The symbols corresponds to the measurement of six different line-strength indices in a sample of 350 stars from the Lick library

Unfortunately, the showed concordance between the analytical formulae and the simulations can not be extrapolated to any general situation. In particular, one of the approximations used to derive Eqs. (11) and (12) is the assumption that the mean signal-to-noise ratio in each 
bandpass can be computed as the quadratic sum of the individual signal-to-noise ratio in each pixel (Eqs. 16-18). In other words

$$
\frac{\left[\sum_{i=1}^{N_{\text {pixels }}} S\left(\lambda_{i}\right)\right]^{2}}{\sum_{i=1}^{N_{\text {pixels }}} \sigma^{2}\left(\lambda_{i}\right)} \approx \sum_{i=1}^{N_{\text {pixels }}} \frac{S^{2}\left(\lambda_{i}\right)}{\sigma^{2}\left(\lambda_{i}\right)}
$$

where $N_{\text {pixels }}$ refers to the number of pixels involved in the measurement of a particular bandpass. The results presented in Fig. 2 were obtained employing data and error spectra in which Eq. (19) worked properly. However, it is straightforward to see that, in this expression, a simultaneous combination of large and small $\sigma(\lambda)$ values would lead to a poor agreement between both terms.

For example, discrepancies between González's formulae and numerical simulations are apparent in the measurement of the $\mathrm{Mg}_{2}$ index in the spectra of the central dominant galaxy of the cluster Abell 2255 (observed with the TWIN spectrograph at the $3.5 \mathrm{~m}$ Telescope of Calar Alto, August 1994), as it is shown in Fig. 3. These differences are due to the fact that a bright sky-line falls within the central bandpass of the $\mathrm{Mg}_{2}$ index. Sky subtraction during the reduction process introduces a larger error in the pixels where sky-lines are present. As a result, the reduced error spectra exhibit, simultaneously, pixels with very different $\sigma(\lambda)$ values, and Eq. (19) is a poor approximation.

\section{The new formulae}

We have derived a more accurate set of analytical formulae to compute errors in line-strength indices. The expected random error in the measurement of an atomic index is given by

$\sigma\left[I_{\mathrm{a}}\right]=\sigma\left[\int_{\lambda_{c_{1}}}^{\lambda_{c_{2}}} \frac{S(\lambda)}{C(\lambda)} \mathrm{d} \lambda\right]$

In practice, this integral must be transformed into the summation

$\sigma\left[I_{\mathrm{a}}\right] \simeq \sigma\left[\Theta \sum_{i=1}^{N_{\text {pixels }}} \frac{S\left(\lambda_{i}\right)}{C\left(\lambda_{i}\right)}\right] \equiv \Theta \sigma[\mathcal{I}]$

where $\Theta$ is the dispersion (in $\AA /$ pixel), assuming a linear wavelength scale, and $N_{\text {pixels }}$ the number of pixels covering the central bandpass (note that, in general, fractions of pixels must be considered in the borders of the bandpasses). $\mathcal{I}$ is a function of $2 \times N_{\text {pixels }}$ variables $\mathcal{I}\left(\ldots, S\left(\lambda_{i}\right), \ldots, C\left(\lambda_{j}\right), \ldots\right)$ which verify

$$
\begin{aligned}
& \operatorname{cov}\left(S\left(\lambda_{i}\right), S\left(\lambda_{j}\right)\right)=0 \forall i, j \in\left[1, N_{\text {pixels }}\right], \quad i \neq j \\
& \operatorname{cov}\left(S\left(\lambda_{i}\right), C\left(\lambda_{j}\right)\right)=0 \forall i, j \in\left[1, N_{\text {pixels }}\right] \\
& \operatorname{cov}\left(C\left(\lambda_{i}\right), C\left(\lambda_{j}\right)\right) \neq 0 \quad \forall i, j \in\left[1, N_{\text {pixels }}\right], \quad i \neq j
\end{aligned}
$$

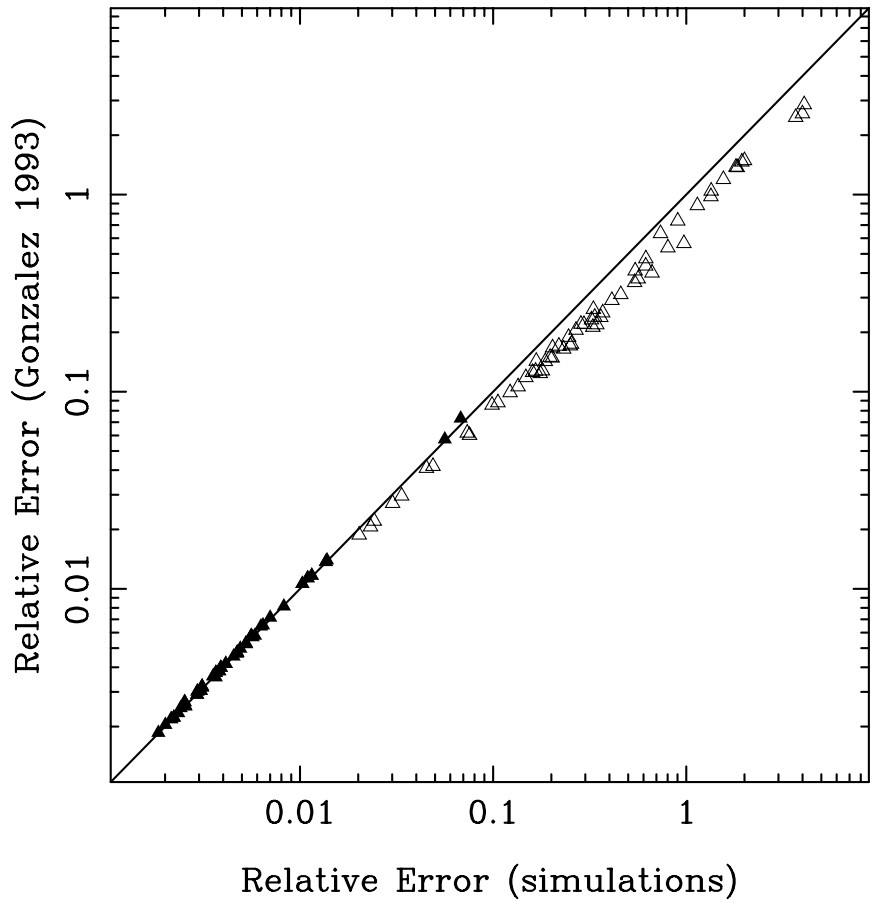

Fig. 3. Comparison of the $\mathrm{Mg}_{2}$ relative errors employing González's formulae and numerical simulations. Filled triangles refer to a sample of 40 bright stars from the Lick Library, whereas open triangles correspond to $\mathrm{Mg}_{2}$ measurements along the radius of the $\mathrm{cD}$ galaxy in Abell 2255. When using González's expressions, there is a clear underestimation of the relative errors in the galaxy due to the subtraction of a bright sky-line in the $\mathrm{Mg}_{2}$ central bandpass during the reduction process

since $C(\lambda)$ is computed from Eq. (5). Taking this result into account:

$\sigma^{2}[\mathcal{I}]=$

$\sum_{i=1}^{N_{\text {pixels }}}\left[\left(\frac{\partial \mathcal{I}}{\partial S\left(\lambda_{i}\right)}\right)^{2} \sigma^{2}\left(\lambda_{i}\right)\right]+\sum_{i=1}^{N_{\text {pixels }}}\left[\left(\frac{\partial \mathcal{I}}{\partial C\left(\lambda_{i}\right)}\right)^{2} \sigma_{C\left(\lambda_{i}\right)}^{2}\right]$

$+\sum_{i=1}^{N_{\text {pixels }}} \sum_{j=1, j \neq i}^{N_{\text {pixels }}}\left[\left(\frac{\partial \mathcal{I}}{\partial C\left(\lambda_{i}\right)}\right)\left(\frac{\partial \mathcal{I}}{\partial C\left(\lambda_{j}\right)}\right) \operatorname{cov}\left(C\left(\lambda_{i}\right), C\left(\lambda_{j}\right)\right)\right]$

$=\sum_{i=1}^{N_{\text {pixels }}}\left[\frac{C^{2}\left(\lambda_{i}\right) \sigma^{2}\left(\lambda_{i}\right)+S^{2}\left(\lambda_{i}\right) \sigma_{C\left(\lambda_{i}\right)}^{2}}{C^{4}\left(\lambda_{i}\right)}\right]$

$+\sum_{i=1}^{N_{\text {pixels }}} \sum_{j=1, j \neq i}^{N_{\text {pixels }}}\left[\left(\frac{S\left(\lambda_{i}\right)}{C^{2}\left(\lambda_{i}\right)}\right)\left(\frac{S\left(\lambda_{j}\right)}{C^{2}\left(\lambda_{j}\right)}\right) \operatorname{cov}\left(C\left(\lambda_{i}\right), C\left(\lambda_{j}\right)\right)\right]$.

After some manipulation the covariance terms are

$\operatorname{cov}\left(C\left(\lambda_{i}\right), C\left(\lambda_{j}\right)\right)=$ 
$\left\langle C\left(\lambda_{i}\right) C\left(\lambda_{j}\right)\right\rangle-\left\langle C\left(\lambda_{i}\right)\right\rangle\left\langle C\left(\lambda_{j}\right)\right\rangle=$

$\Lambda_{1} \sigma_{S_{b}}^{2}+\Lambda_{2} \operatorname{cov}\left(S_{b}, S_{r}\right)+\Lambda_{3} \operatorname{cov}\left(S_{r}, S_{b}\right)+\Lambda_{4} \sigma_{S_{r}}^{2}$

where we have defined the following four parameters

$$
\begin{aligned}
\Lambda_{1} & \equiv \frac{\left(\lambda_{r}-\lambda_{i}\right)\left(\lambda_{r}-\lambda_{j}\right)}{\left(\lambda_{r}-\lambda_{b}\right)^{2}} \\
\Lambda_{2} & \equiv \frac{\left(\lambda_{r}-\lambda_{i}\right)\left(\lambda_{j}-\lambda_{b}\right)}{\left(\lambda_{r}-\lambda_{b}\right)^{2}} \\
\Lambda_{3} & \equiv \frac{\left(\lambda_{i}-\lambda_{b}\right)\left(\lambda_{r}-\lambda_{j}\right)}{\left(\lambda_{r}-\lambda_{b}\right)^{2}} \\
\Lambda_{4} & \equiv \frac{\left(\lambda_{i}-\lambda_{b}\right)\left(\lambda_{j}-\lambda_{b}\right)}{\left(\lambda_{r}-\lambda_{b}\right)^{2}} .
\end{aligned}
$$

Since $S_{b}$ y $S_{r}$ are not correlated, we obtain

$\operatorname{cov}\left(C\left(\lambda_{i}\right), C\left(\lambda_{j}\right)\right)=\Lambda_{1} \sigma_{S_{b}}^{2}+\Lambda_{4} \sigma_{S_{r}}^{2}$

and finally

$$
\begin{aligned}
& \frac{\sigma^{2}\left[I_{\mathrm{a}}\right]}{\Theta^{2}}=\sum_{i=1}^{N_{\text {pixels }}}\left[\frac{C^{2}\left(\lambda_{i}\right) \sigma^{2}\left(\lambda_{i}\right)+S^{2}\left(\lambda_{i}\right) \sigma_{C\left(\lambda_{i}\right)}^{2}}{C^{4}\left(\lambda_{i}\right)}\right] \\
& +\sum_{i=1}^{N_{\text {pixels }}} \sum_{j=1, j \neq i}^{N_{\text {pixels }}}\left[\frac{S\left(\lambda_{i}\right) S\left(\lambda_{j}\right)}{C^{2}\left(\lambda_{i}\right) C^{2}\left(\lambda_{j}\right)}\left(\Lambda_{1} \sigma_{S_{b}}^{2}+\Lambda_{4} \sigma_{S_{r}}^{2}\right)\right]
\end{aligned}
$$

where

$\sigma_{C\left(\lambda_{i}\right)}^{2}=\left(\frac{\lambda_{r}-\lambda_{i}}{\lambda_{r}-\lambda_{b}}\right)^{2} \sigma_{S_{b}}^{2}+\left(\frac{\lambda_{i}-\lambda_{b}}{\lambda_{r}-\lambda_{b}}\right)^{2} \sigma_{S_{r}}^{2}$

$\sigma_{S_{b}}^{2}=\frac{\Theta^{2}}{\left(\lambda_{b_{2}}-\lambda_{b_{1}}\right)^{2}} \sum_{i=1}^{N_{\text {pixels(blue) }}} \sigma^{2}\left(\lambda_{i}\right)$

$\sigma_{S_{r}}^{2}=\frac{\Theta^{2}}{\left(\lambda_{r_{2}}-\lambda_{r_{1}}\right)^{2}} \sum_{i=1}^{N_{\text {pixels(red) }}} \sigma^{2}\left(\lambda_{i}\right)$.

Errors in the molecular indices are calculated through

$\sigma\left[I_{\mathrm{m}}\right]=2.5 \frac{\log _{10} \mathrm{e}}{10^{-0.4 I_{\mathrm{m}}}} \frac{1}{\lambda_{c_{2}}-\lambda_{c_{1}}} \sigma\left[I_{\mathrm{a}}\right]$

which is identical to Eq. (12).

Using the new formulae, the computed index errors for the samples presented in Figs. 2 and 3 completely agree with the results from numerical simulations (see Fig. 4).

The errors in the $4000 \AA$ break, defined in Eq. (8), can be computed as

$\sigma^{2}\left[D_{4000}\right]=\frac{\mathcal{F}_{r}^{2} \sigma_{\mathcal{F}_{b}}^{2}+\mathcal{F}_{b}^{2} \sigma_{\mathcal{F}_{r}}^{2}}{\mathcal{F}_{b}^{4}}$

where

$\mathcal{F}_{p} \equiv \Theta \sum_{i=1}^{N_{\text {pixels }}}\left[\begin{array}{ll}\lambda_{i}^{2} & S\left(\lambda_{i}\right)\end{array}\right]$

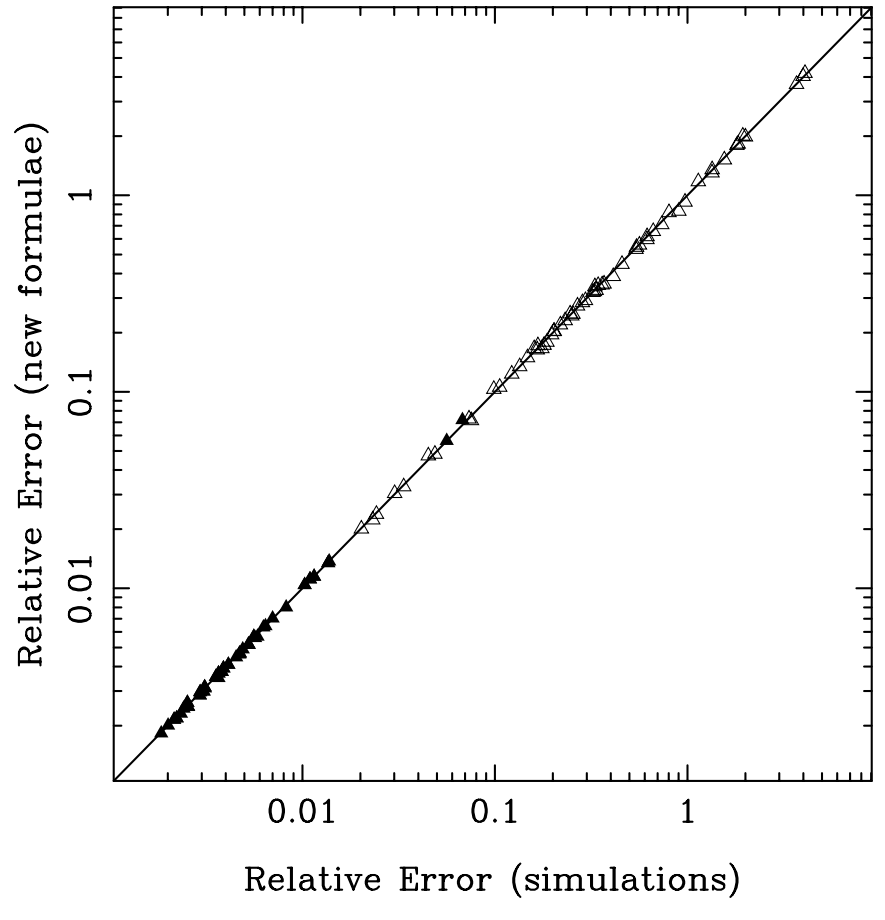

Fig. 4. Comparison of the $\mathrm{Mg}_{2}$ relative errors employing the new formulae and numerical simulations for the samples shown in Fig. 3. The agreement between both methods is complete

$\sigma_{\mathcal{F}_{p}}^{2}=\Theta^{2} \sum_{i=1}^{N_{\text {pixels }}}\left[\lambda_{i}^{4} \sigma^{2}\left(\lambda_{i}\right)\right]$

(the subscript $p$ refers indistinctly to $b$ or $r$ ). Note that in this case $\mathcal{F}_{b}$ and $\mathcal{F}_{r}$ are not correlated. $D_{4000}$ errors computed in this way show a perfect agreement with numerical simulations.

\section{Estimation of $S / N$ ratios}

As it is apparent from Fig. 1, there is a clear correlation between the measured relative error and the $(S / N$ ratio)/A. Although such a correlation arises naturally, quantitative estimates of errors as a function of $(S / N$ ratio) $/ \AA$ are also expected to depend on index values.

We have shown that the use of approximate formulae for the computation of errors can lead to misleading error estimates. However, performing appropriate simplifications, it is possible to derive simple expressions to estimate the absolute index error as a function of the mean $(S / N$-ratio $) / \AA$. It can be shown that

$\sigma\left[I_{\mathrm{a}}\right] \approx \frac{c_{1}-c_{2} I_{\mathrm{a}}}{S N(\AA)}$

where, to simplify, we have defined $S N(\AA)$ as

$S N(\AA)=\frac{1}{N \sqrt{\Theta}} \sum_{i=1}^{N} \frac{S\left(\lambda_{i}\right)}{\sigma\left(\lambda_{i}\right)}$ 
(the summation extends over the three bandpasses, i.e. $N$ pixels). The two constants $c_{1}$ and $c_{2}$ are defined as follows

$c_{1} \equiv \Delta \lambda_{c} c_{2}$

$c_{2} \equiv \sqrt{\frac{1}{\Delta \lambda_{c}}+\left(\frac{\lambda_{r}-\lambda_{c}}{\lambda_{r}-\lambda_{b}}\right)^{2} \frac{1}{\Delta \lambda_{b}}+\left(\frac{\lambda_{c}-\lambda_{b}}{\lambda_{r}-\lambda_{b}}\right)^{2} \frac{1}{\Delta \lambda_{r}}}$

being $\Delta \lambda_{b}, \Delta \lambda_{c}$ and $\Delta \lambda_{r}$ the bandpass widths.

Similarly

$\sigma\left[I_{\mathrm{m}}\right] \approx \frac{c_{3}}{S N(\AA)}$

where

$c_{3} \equiv 2.5 c_{2} \log _{10} \mathrm{e}$.

Equations (41) and (45) can be easily employed to predict the required $(S / N$-ratio) $/ \AA$ to achieve a fixed index error. In Fig. 5 we show the predictions of these expressions for some particular indices compared with the actual error measurements (from Eqs. (33) and (37)) in a star sample. It is interesting to note that the absolute error of molecular indices does not depend, in a first approximation, on the absolute index value, although the contrary is true for the atomic indices. This is the reason why a larger scatter is apparent in panel (a), where $I_{\mathrm{a}}$ in Eq. (41) has been replaced by its corresponding arithmetic mean in the sample. Numerical values for $c_{1}, c_{2}$ and $c_{3}$, which obviously depend on the considered index, are given in Table 1.

Following the same procedure with the $D_{4000}$ index:

$\sigma\left[D_{4000}\right] \approx \frac{D_{4000}}{\sqrt{200}} \sqrt{\frac{1}{S N(\AA)_{b}^{2}}+\frac{1}{S N(\AA)_{r}^{2}}}$

where $S N(\AA)_{b}$ and $S N(\AA)_{r}$, the mean $(S / N$-ratio $) / \AA$ in the blue and red band respectively, will attain, in general, different values (given the large wavelength coverage of the break). In this case, the relative $D_{4000}$ error does not depend on the absolute $D_{4000}$ value.

\section{Summary}

We present a set of analytical formulae to compute random errors in the measurement of line-strength indices. The new expressions constitute an improvement compared with the approaches followed in previous works, providing a straight method to attain reliable random error estimates. In particular, the derived equations should be preferred to methods involving either numerical simulations (a computer time demanding method) or multiple observations (usually prohibitive at low light levels). As a useful tool for observation planning, we also provide simple recipes to estimate the required signal-to-noise ratio to achieve a desired index error.

We want to stress here that full benefit from these formulae can only be obtained after a proper treatment

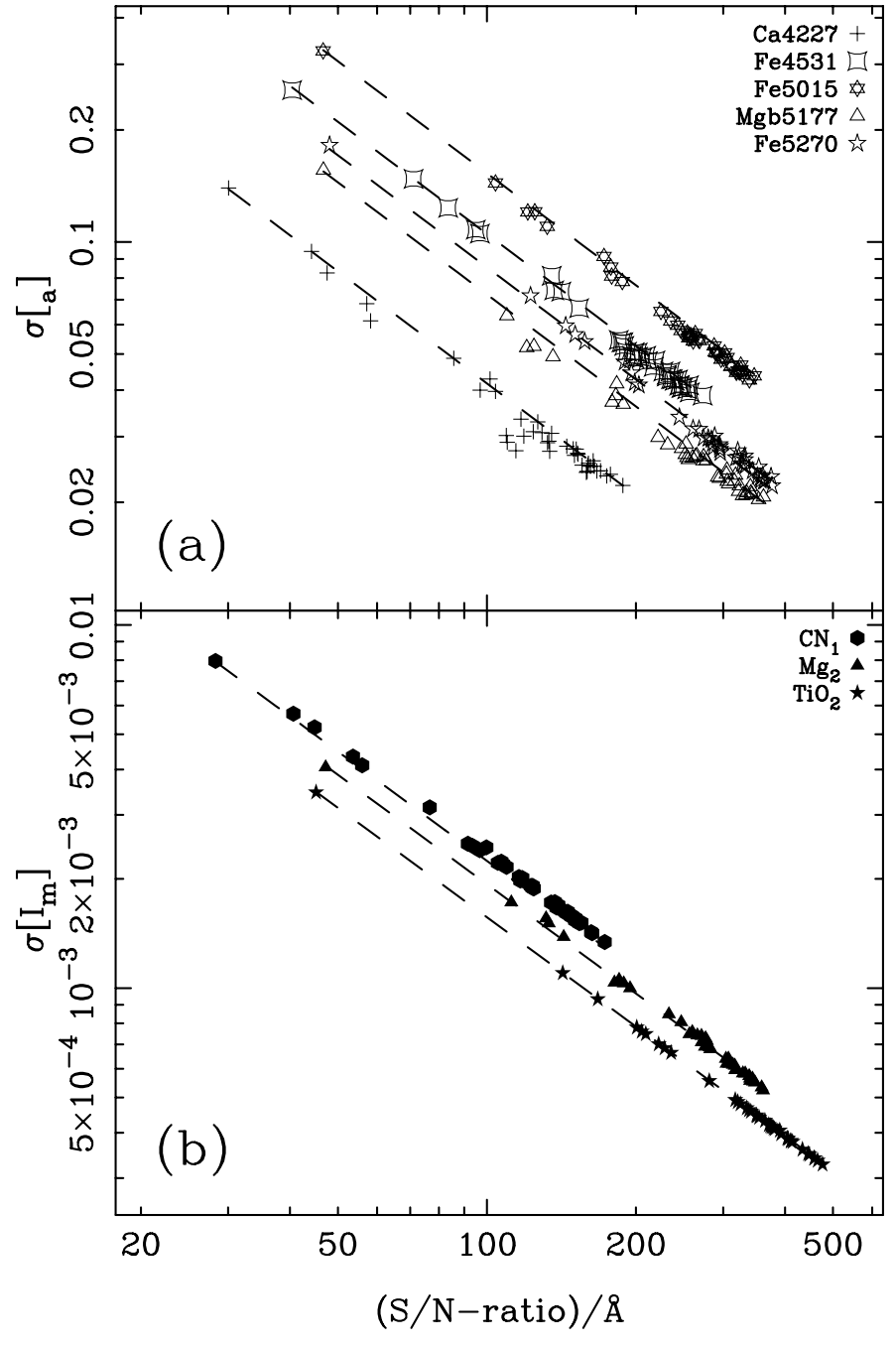

Fig. 5. Absolute atomic (panel a)) and molecular (panel b)) line-strength errors measured in the sample of 40 stars from the Lick library as a function of the mean $(S / N$-ratio)/ $\AA$. The predictions of Eqs. (41) and (45) are plotted as dashed lines

of the error propagation throughout the data reduction. Apart from this, the analytical method followed to derived the expressions given in Sect. 6 is exact, in the sense that it does not involve any approximation (exception made for the unavoidable data sampling).

Acknowledgements. We are grateful to the referee for useful comments. The JKT is operated on the island of La Palma by the Royal Greenwich Observatory at the Observatorio del Roque de los Muchachos of the Instituto de Astrofísica de Canarias. The Calar Alto Observatory is operated jointly by the Max-Planck-Institute für Astronomie, Heidelberg, and the Spanish Comisión Nacional de Astronomía. This work was supported by the Spanish "Programa Sectorial de Promoción General del Conocimiento" under grant No. PB96-0610. 


\section{References}

Bender R., Surma P., 1992, A\&A 258, 250

Bender R., Ziegler B., Bruzual G., 1996, ApJ 463, L51

Bica E., Alloin D., Schmidt A.A., 1990, A\&A 228, 33

Bressan A., Chiosi C., Tantalo R., 1996, A\&A 311, 425

Brodie J.P., Hanes D.A., 1986, ApJ 300, 258

Brodie J.P., Huchra J.P., 1990, ApJ 362, 503

Bruzual A.G., 1983, ApJ 273, 105

Burstein D., Faber S.M., Gaskell C.M., Krumm N., 1984, ApJ 287, 586

Burstein D., Faber S.M., González J.J., 1986, AJ 91, 1130

Cardiel N., Gorgas J., 1997 (in preparation)

Cardiel N., Gorgas J., Aragón-Salamanca A., 1995, MNRAS 277,502

Carollo C.M., Danziger I.J., Buson L., 1993, MNRAS 265, 553

Charlot S., Silk J., 1994, ApJ 432, 453

Couture J., Hardy E., 1988, AJ 96, 867

Davidge T.J., 1992, AJ 103, 1512

Davies R.L., 1996, The Star Formation History of Elliptical Galaxies. In: Buzzoni A., Renzini A., Serrano A. (eds.) ASP Conf. Ser. 86, Fresh Views of Elliptical Galaxies, p. 175

Davies R.L., Sadler E.M., Peletier R.F., 1993, MNRAS 262, 650

Díaz A.I., Terlevich E., Terlevich R., 1989, MNRAS 239, 325

Dressler A., Lynden-Bell D., Burstein D., et al., 1987, ApJ 313, 42

Faber S.M., 1973, ApJ 179, 731

Faber S.M., Burstein D., Dressler A., 1977, AJ 82, 941

Faber S.M., Friel E.D., Burstein D., Gaskell C.M., 1985, ApJS 57,711

Fisher D., Franx M., Illingworth G., 1995, ApJ 448, 119

Fisher D., Franx M., Illingworth G., 1996, ApJ 459, 110

Gilliland R.L., 1992, Details of Noise Sources and Reduction Processes. In: Howell S.B. (ed.) ASP Conf. Ser. 23, Astronomical CCD Observing and Reduction Techniques, p. 68
González J.J., 1993, Ph.D. thesis, University of California, Santa Cruz

Goudfrooij P., Emsellem E., 1996, A\&A 306, L45

Gorgas J., 1987, Ph.D. thesis, Universidad Complutense, Madrid

Gorgas J., Efstathiou G., Aragón-Salamanca A., 1990, MNRAS 245, 217

Gorgas J., Faber S.M., Burstein D., et al., 1993, ApJS 86, 153

Gregg M.D., 1994, AJ 108, 2164

Hamilton D., 1985, ApJ 297, 371

Huchra J.P., Brodie J.P., Caldwell N., Christian C., Shommer R., 1996, ApJS 102, 29

Jones L.A., Worthey G., 1995, ApJ 446, L31

Minniti D., 1995, A\&AS 113, 299

Mould J.R., Oke J.B., de Zeeuw P.T., Nemec J.M., 1990, AJ 99, 1823

Rich R.M., 1988, AJ 95, 828

Rose J.A., 1994, AJ 107, 206

Saglia R.P., Bertin G., Bertola F., et al., 1993, ApJ 567, 572

Schweizer F., Seitzer P., Faber S.M., et al., 1990, ApJ 364, L33

Terndrup D.M., Sadler E.M., Rich R.M., 1995, AJ 110, 1774

Thomsen B., Baum W.A., 1987, ApJ 315, 460

Trager S.C., 1997, Ph.D. thesis, University of California, Santa Cruz

Vazdekis A., Casuso E., Peletier R.F., Beckman J.E., 1996, ApJS 106, 307

Vazdekis A., Peletier R.F., Beckman J.E., Casuso E., 1997, ApJS (in press)

Whitford A.E., Rich R.M., 1983, ApJ 274, 723

Worthey G., 1994, ApJS 95, 107

Worthey G., Faber S.M., González J.J., 1992, ApJ 398, 69

Worthey G., Faber S.M., González J.J., Burstein D., 1994, ApJS 94, 687

Zhou X., 1991, A\&A 248, 367 